\title{
Mulheres no pós-alta de hanseníase: aspectos clínicos, sociodemográficos e
}

\section{reprodutivos}

\author{
Women in post-high leprosy: clinical, sociodemographic and reproductive aspects \\ Mujeres em post-alta lepra: aspectos clínicos, sociodemográficos y reproductivos
}

Recebido: 19/12/2020 | Revisado: 27/12/2020 | Aceito: 03/01/2021 | Publicado: 05/01/2021

\author{
Ana Beatriz de Almeida Lima \\ ORCID: https://orcid.org/0000-0001-6588-1070 \\ Universidade de São Paulo, Brasil \\ E-mail: ana.beatriz_al@usp.br \\ Clodis Maria Tavares \\ ORCID: https://orcid.org/0000-0001-6804-3064 \\ Universidade Federal de Alagoas, Brasil \\ E-mail: clodistavares@yahoo.com.br \\ Igor Michel Ramos dos Santos \\ ORCID: https://orcid.org/0000-0001-6557-3369 \\ Universidade Federal de Alagoas, Brasil \\ E-mail: igor.santos@esenfar.ufal.br \\ Gabriela Rodrigues Bragagnollo \\ ORCID: https://orcid.org/0000-0003-1480-8046 \\ Universidade de São Paulo, Brasil \\ E-mail: gabriela.rodrigues.bragagnollo@usp.br \\ Nataly Mayara Cavalcante Gomes \\ ORCID: https://orcid.org/0000-0001-9334-7887 \\ Universidade Estadual de Ciências da Saúde de Alagoas, Brasil \\ E-mail: natallymayara12@gmail.com \\ Amuzza Aylla Pereira dos Santos \\ ORCID: https://orcid.org/0000-0001-6299-7190 \\ Universidade Federal de Alagoas, Brasil \\ E-mail: amuzza.santos@gmail.com
}

\begin{abstract}
Resumo
Objetivo: Analisar as variáveis referentes aos aspectos sociodemográficos, clínicos e reprodutivos das mulheres pósalta de hanseníase. Método: Trata-se de um estudo analítico transversal com abordagem quantitativa, na qual construiu-se um instrumento que continham dados referentes aos aspectos sociodemográficos e história reprodutiva de mulheres pós-alta de hanseníase durante as consultas de enfermagem em Unidades Básicas de Saúde (UBS) do município de Rio Largo, Alagoas, no período de abril a julho de 2019. Resultados: As características demográficas e reprodutivas da população estudada foram: mulheres predominantemente pardas de baixa escolaridade (fundamental incompleto) e analfabetas, com idade acima dos 55 anos, que desempenham funções do lar e com renda de 1 salário mínimo e meio, em relação aos aspectos clínicos $38,1 \%$ foram diagnosticadas com forma clínica indeterminada e 66,7\% paucibacilares. Conclusão: Observou-se a necessidade de um acompanhamento especial para as mulheres no pós-alta da hanseníase, uma vez que a grande maioria destas mulheres se encontram em idade reprodutiva. É essencial promover um cuidado em busca de prepará-las para uma prática anticonceptiva segura e a prevenção de uma gestação de alto risco.
\end{abstract}

Palavras-chave: Hanseníase; Saúde da mulher; Doenças negligenciadas; Saúde reprodutiva; Enfermagem.

\begin{abstract}
Objective: To analyze the variables related to the sociodemographic, clinical and reproductive aspects of postdischarge women for leprosy. Method: This is a cross-sectional analytical study with a quantitative approach, in which an instrument was constructed that contained data referring to the sociodemographic aspects and reproductive history of post-discharge women for leprosy during nursing consultations in Basic Health Units (UBS) from the county of Rio Largo, Alagoas, from April to July 2019. Results: The demographic and reproductive characteristics of the studied population were: predominantly brown women with low education (incomplete elementary) and illiterate, aged over 55 years, who perform household duties and with an income of 1 and a half minimum wages, in relation to clinical aspects $38.1 \%$ were diagnosed with undetermined clinical form and $66.7 \%$ paucibacillary. Conclusion: It was observed a need for special monitoring for women after leprosy discharge, since the vast majority of these women are of reproductive age. It is essential to promote care in order to prepare them for safe contraceptive practice and the prevention of high-risk pregnancies.
\end{abstract}

Keywords: Leprosy; Womens's health; Neglected diseases; Reproductive health; Nursing. 


\section{Resumen}

Objetivo: Analizar las variables relacionadas con los aspectos sociodemográficos, clínicos y reproductivos de las mujeres post alta por la lepra. Método: Se trata de un estudio analítico transversal con enfoque cuantitativo, en el cual se construyó un instrumento que contenía datos referentes a los aspectos sociodemográficos y antecedentes reproductivos de mujeres post alta por la lepra durante las consultas de enfermería en Unidades Básicas de Salud (UBS) del municipio de Rio Largo, Alagoas, de abril a julio de 2019. Resultados: Las características demográficas y reproductivas de la población estudiada fueron: predominantemente mujeres morenas con baja escolaridad (primaria incompleta) y analfabetas, mayores de 55 años, que realizan funciones domésticas y con un ingreso de 1 salario mínimo y medio, en relación a aspectos clínicos se diagnosticó forma clínica indeterminada al 38,1\% y paucibacilar al 66,7\%. Conclusión: Se observo la necesidad de un seguimiento especial de las mujeres después del alta por lepra, ya que la gran mayoría de estas mujeres se encuentran en edad reproductiva. Es fundamental promover los cuidados para prepararlas para la práctica anticonceptiva segura y la prevención de embarazos de alto riesgo.

Palabras clave: Lepra, Salud da mujer; Enfermedades desatendidas; Salud reproductiva; Enfermaría.

\section{Introdução}

A hanseníase caracteriza-se como uma doença negligenciada de histórico milenar, no entanto, apesar de ser uma doença curável um dos grandes desafios para o enfrentamento consiste na questão social e cultura do indivíduo, uma vez que as pessoas mais afetadas são oriundas de países em desenvolvimento, mostrando assim uma relação direta entre desigualdade social e de gênero, condições de vida e saúde e a incidência/prevalência da doença (Gonçalves et al., 2018; Lopes \& Rangel, 2014; Monte \& Pereira, 2015).

É notório que homens e mulheres manifestam necessidades diferentes quanto às questões referentes à saúde, não apenas relacionadas a situações específicas, bem como, ao acesso à proteção de saúde. Sendo assim, o processo de adoecimento pode causar alterações nos núcleos familiares e nos casos de hanseníase no sexo feminino, percebe-se que a mulher pode sofrer discriminação em dose dupla tanto pelo gênero quanto pela doença (Monte \& Pereira, 2015).

Nesse contexto, observa-se que nos países em desenvolvimento, a maioria das mulheres são donas de casa negligenciadas dentro da família, especialmente no que diz respeito à saúde, gerando impacto no seu bem-estar e de seus familiares. Acrescido a isso, tem-se as questões reprodutiva, social, e do ciclo gravídico puerperal (Sarkar \& Pradhan, 2016).

A hanseníase permanece com uma doença de importância epidemiológica e social a nível nacional e internacional, estudos que investigam as suas manifestações no sexo feminino, englobando os fatores que determinam e condicionam o processo saúde-doença são necessários para auxiliar na compreensão do impacto dessa enfermidade neste público e colaborar em uma assistência pautada no princípio da integralidade do cuidado. Desta forma, o objetivo deste estudo é analisar as variáveis referentes aos aspectos sociodemográficos, clínicos e reprodutivos das mulheres pós-alta de hanseníase.

\section{Metodologia}

Trata-se de um estudo analítico transversal com abordagem quantitativa, no qual construiu-se um instrumento que continham dados referentes aos aspectos sociodemográficos e história reprodutiva de mulheres pós-alta de hanseníase durante as consultas de enfermagem em Unidades Básicas de Saúde (UBS) do município de Rio Largo, Alagoas, no período de abril a julho de 2019.

Os estudos analíticos estão relacionados a distribuição de eventos na população, estimando incidência e prevalência. Onde se introduz um fator de exposição ou de causalidade, e avalia-o através de ferramentas estatísticas. Já o estudo transversal é o "retrato" da situação no momento em que o pesquisador observa. A população ou amostra é estudada em um único momento, utilizado para avaliar se existe relação entre as variáveis da pesquisa e é importante para determinação de prevalência de doenças, é considerado um estudo barato e fácil de ser realizado e pode gerar hipóteses de associação ao risco (Capp \& Nienov, 2020). 
O instrumento foi adaptado com informações da ficha de notificação e investigação, além de referenciais da área de enfermagem e saúde reprodutiva. Incluiu-se na investigação pacientes do sexo feminino maiores de 15 anos, residente no município de Rio Largo, que foram diagnosticadas com hanseníase durante o período de 2007 a 2017 no SINAN.

Durante o período delimitado neste estudo, o município notificou 46 mulheres afetadas pela hanseníase. Dentre essas, 01 teve diagnóstico errado, 02 foram a óbito, 03 encontravam-se em outro município, 03 recusaram-se a participar do estudo e 16 não foram localizadas na cidade. A amostra final foi constituída por 21 mulheres no pós-alta em hanseníase. A aplicação do instrumento se deu por meio da consulta de enfermagem realizada nas UBS ou durante as visitas domiciliares ao público-alvo.

Os dados coletados se referem à idade, raça/cor, escolaridade, renda, ocupação, estado civil, gestações, partos anteriores, número de filhos, amamentou, engravidou no percurso da doença, estava grávida no momento do diagnóstico, realizou consulta de pré-natal, exame preventivo (Papanicolau) e mamografia. Sendo tabulados em uma planilha do Microsoft Office Excel® 2016 e posteriormente processado e realizada análise estatística descritiva no programa Statistical Package for the Social Sciences (SPSS), versão 21.0.

O projeto de pesquisa foi apreciado pelo Comitê de Ética em Pesquisa da Universidade Federal de Alagoas, aprovado sob parecer de Certificado de Apresentação para Apreciação Ética (CAAE) n02327118.4.0000.5013. Foram respeitadas as normas éticas previstas na Resolução do Conselho Nacional de Saúde no 466/12 e 510/2016, garantindo a manutenção da integridade, a privacidade e o sigilo das informações das participantes do estudo.

\section{Resultados}

Ao analisar as variáveis sociodemográficas, observou que o predomínio da faixa etária está acima de 45 anos correspondendo a $42,9 \%$, constituído por mulheres em sua maioria pardas $(52,5 \%)$, donas de casa $(57,1 \%)$, analfabetas $(28,6 \%)$ e 33,3\% com ensino fundamental incompleto, conforme é possível perceber na Tabela 1. 
Tabela 1 - Distribuição dos dados sociodemográficos de mulheres pós-alta de Hanseníase no período de 2007 a 2017 no município de Rio Largo-AL, Brasil, 2019 (n=21).

\begin{tabular}{|c|c|c|c|}
\hline Variáveis & Categorias & $\mathbf{n}$ & $\%$ \\
\hline \multirow[t]{5}{*}{ Idade } & Entre 18 e 25 anos & 2 & 9,5 \\
\hline & Entre 26 e 35 anos & 2 & 9,5 \\
\hline & Entre 36 e 45 anos & 4 & 19 \\
\hline & Entre 46 e 55 anos & 4 & 19 \\
\hline & Acima de 55 anos & 9 & 43 \\
\hline \multirow[t]{5}{*}{ Ocupação } & Aposentada & 3 & 14,3 \\
\hline & Dona de casa & 12 & 57,1 \\
\hline & Autônoma & 3 & 14,3 \\
\hline & Diarista & 2 & 9,5 \\
\hline & Recepcionista & 1 & 4,8 \\
\hline \multirow[t]{4}{*}{ Cor } & Branca & 4 & 19 \\
\hline & Preta & 4 & 19 \\
\hline & Amarela & 2 & 9,5 \\
\hline & Parda & 11 & 52,5 \\
\hline \multirow[t]{5}{*}{ Escolaridade } & Analfabeta & 6 & 28,6 \\
\hline & Ensino Fundamental I & 7 & 33,3 \\
\hline & $\left(1^{\circ}\right.$ ao $5^{\circ}$ ano $)$ & & \\
\hline & $\begin{array}{l}\text { Ensino Fundamental II } \\
\left(6^{\circ} \text { ao } 9^{\circ} \text { ano }\right)\end{array}$ & 3 & 14,3 \\
\hline & Ensino Médio & 5 & 23,8 \\
\hline \multirow[t]{2}{*}{ Renda } & Até 1 salário mínimo e meio & 19 & 90,5 \\
\hline & Acima de 1 salário mínimo e meio & 2 & 9,5 \\
\hline
\end{tabular}

Fonte: Autores (2019).

Das 21 mulheres entrevistadas, 38,1\% foram diagnosticadas com a forma clínica indeterminada, sobretudo paucibacilares $(66,7 \%)$, onde $67 \%$ no momento do diagnóstico foram classificadas com grau 0 de incapacidade física e $43 \%$ não possuíam o item sobre grau de incapacidade no momento da cura, tendo expressividade a alta por cura, conforme exposto nas Tabelas 2 e 3 . 
Tabela 2 - Dados clínicos segundo classificação de Madri e classificação operacional, das mulheres pós-alta de Hanseníase no período de 2007 a 2017 no município de Rio Largo-AL, Brasil, 2019 (n=21).

\begin{tabular}{llll}
\hline \multicolumn{1}{c}{ Variáveis } & Categorias & n & $\%$ \\
\hline Classificação de Madri & Indeterminada & 8 & 38,1 \\
& Dimorfa & 4 & 19 \\
& Tuberculoide & 5 & 23,9 \\
& Não classificado & 4 & 19 \\
\hline Classificação operacional & Paucibacilar & 14 & 66,7 \\
& Multibacilar & 7 & 33,3 \\
\hline
\end{tabular}

Fonte: Autores (2019).

Tabela 3 - Dados clínicos segundo grau de incapacidade no momento do diagnóstico e alta, e tipo de saída das mulheres pósalta de Hanseníase no período de 2007 a 2017 no município de Rio Largo-AL, Brasil, 2019 (n=21).

\begin{tabular}{llll}
\hline Variáveis & Categorias & n & $\%$ \\
\hline Grau de incapacidade física no momento & Grau 0 & 14 & 67 \\
do diagnóstico & Grau I & 4 & 19 \\
& Grau II & 1 & 5 \\
& Não avaliado & 2 & 9 \\
\hline Grau de incapacidade física no momento & Grau 0 & 9 & 43 \\
da alta & Grau I & 2 & 9 \\
& Não avaliado & 10 & 48 \\
\hline Tipo de saída & Cura & 20 & 95,3 \\
\hline
\end{tabular}

Fonte: SINAN (2019).

Sobre a caracterização atual da história reprodutiva, 66,6\% tiveram de 1 a 4 gestações, dezessete (17) multíparas (mais de um filho). Sobre a idade do primeiro parto, 52,4\% tiveram filhos entre 15 e 19 anos. Duas (2) engravidaram no percurso da doença, onde os sinais de suspeição para Hanseníase foram identificados durante as consultas de pré-natal, sendo a mesma encaminhada à consulta dermatológica, em conformidade com a Tabela 4. Nenhuma delas apresentaram reação hansênica durante a gravidez, puerpério e lactação. 
Tabela 4 - Distribuição dos dados da história reprodutiva das mulheres pós-alta de Hanseníase no período de 2007 a 2017 no município de Rio Largo-AL, Brasil, 2019 (n=21).

\begin{tabular}{llccc}
\hline Variável & Categorias & n & \% \\
\hline Número de gestações & Nenhuma & 1 & 4,8 \\
& 1 a 4 gestações & 14 & 66,6 \\
& 5 a 9 gestações & 5 & 23,8 \\
& Acima de 9 & 1 & 4,8 \\
\hline Estava grávida no momento do diagnóstico & gestações & & \\
\hline Engravidou no percurso da doença & Sim & 2 & 9,5 \\
& Não & 19 & 90,5 \\
\hline Amamentou & Sim & 2 & 9,5 \\
& Não & 19 & 90,5 \\
\hline
\end{tabular}

Fonte: Autores (2019).

Sobre a realização da mamografia, ao serem entrevistadas, três (3) mulheres autorrelataram terem realizado o exame em 2019, seis (6) em 2018, duas (2) em 2017, duas (2) em 2009, uma (1) não recordava, sete (7) não responderam. No tocante a ter realizado o exame preventivo (Papanicolau), duas (2) autorrelatam em 2019, cinco (5) em 2018, duas (2) em 2017, duas (2) em 2016, uma (1) em 2013, uma (1) em 2009. Sendo expressiva a distribuição do exame de Papanicolau na faixa etária de 19 a $59(78,6 \%)$, como pode ser vista na Tabela 5.

Tabela 5 - Distribuição da realização de mamografia e papanicolau por faixa etária mulheres pós-alta de Hanseníase no período de 2007 a 2017 no município de Rio Largo-AL, Brasil, 2019 (n=21).

\begin{tabular}{lllllllll}
\hline & \multicolumn{3}{l}{ Mamografia } & \multicolumn{3}{l}{ Papanicolau } \\
Faixa etária & SIM & NÃO & SIM & NÃO \\
\hline & n & $\%$ & n & $\%$ & n & $\%$ & n & $\%$ \\
\hline & & & & & & & & \\
$19-59$ & 7 & 50 & 7 & 50 & 11 & 78,6 & 3 & 21,4 \\
$60-73$ & 7 & 100 & 0 & 0 & 5 & 71,4 & 2 & 28,6 \\
\hline
\end{tabular}

Fonte: Autores (2019).

\section{Discussão}

A análise das variáveis sociodemográficas e reprodutivas foram capazes de identificar o perfil das mulheres no pósalta de hanseníase. Em relação às características das mulheres que participaram do estudo, estas foram de uma forma geral, semelhantes às da maioria da população brasileira (Souza et al., 2018; Gonçalves et al., 2018; Monteiro et al., 2014). No que 
tange a raça/cor, a maioria se autodeclararam pardas, com isso observa-se uma singularidade com dados nacionais, pois com relação às cinco regiões do Brasil, a proporção de pessoas diagnosticadas com hanseníase na população parda foi mais frequente em 04 das 05 regiões, exceto, a região sul que é predominantemente de indivíduos autodeclarados brancos (Brasil, 2020a).

Um estudo realizado em Arapiraca- AL, os autores apresentaram 201 (68,8\%) dos participantes como morenos e apenas $24(8,2 \%)$ como negros (Albuquerque et al., 2020). Neste quesito, também é possível verificar a semelhança com outro estudo, e traz a reflexão de que esta doença acomete os grupos populacionais mais vulneráveis, e isso é visto como uma prioridade para o desenvolvimento de ações em saúde que visem reduzir as disparidades étnico-raciais nas morbidades por doenças transmissíveis dentro da Política Nacional de Saúde Integral da População Negra (Brasil, 2017a).

Vinculado a isso, encontra-se que essa doença é mais frequente entre pessoas com menor grau de escolaridade, e esta variável é um indicador de condições de vida, o que reforça também o caráter de negligência (Cabral-Miranda, Chiaravalloti \& Barrozo, 2014; Souza et al., 2018). A pouca escolaridade, além de ser indicativo de baixo poder aquisitivo, perpassa por outras dificuldades como a compreensão e incorporação de aspectos importantes relacionados a práticas de educação em saúde e autocuidado, elementos essenciais para controle da doença (Brasil, 2017b; WHO, 2016). Do mesmo modo, receber até um salário mínimo pode ser uma condição relacionada a dificuldades de acesso a serviços de saúde assim como, para o controle da dinâmica de doenças negligenciadas (Boigny et al., 2019; Mieras et al., 2015).

Coincidente a isso, estados da região Nordeste, marcados por grande desigualdade social, apresentam maior expressão dos impactos de tais endemias na saúde das populações mais vulneráveis (Souza et al., 2018; Martins-Melo et al., 2016). Desta forma, reforça-se que a hanseníase é mais frequente entre os menos favorecidos, seja por motivos de educação em saúde, moradia, saneamento básico, entre outros (Gonçalves et al., 2018).

Com relação, as participantes desse estudo, em sua maioria, encontram-se na fase economicamente ativa, apesar de $57,1 \%$ das participantes terem como ocupação o cuidado com o lar. Sabe-se que ter meios que proporcionem geração de renda, reflete diretamente na autonomia e na maneira como a pessoa conduz as decisões de sua vida. No que se refere, a associação desse período ativo para o mercado de trabalho um estudo realizado no interior da cidade de São Paulo entrevistou mulheres acometidas pela hanseníase com o objetivo de analisar a interferência da doença na relação do trabalho e da vida diária, apontou que as alterações na rotina do trabalho são nítidos, mesmo que velado, e as evidências são mais perceptíveis e sensíveis nos indivíduos que foram acometidos por alguma incapacidade física, mas sem desconsiderar os aspectos biopsicossocial (Gonçalves et al., 2018).

Constatou-se que as profissões e as ocupações desenvolvidas pelas participantes demandam esforço físico. Tal fato pode contribuir, no futuro, para um aumento da proporção de mulheres inativas, considerando a natureza crônica e incapacitante da doença. Ainda, verificou-se maior proporção de casos paucibacilares na população estudada, o que indica o diagnóstico oportuno nos serviços de saúde local. Isso pode estar relacionado com fatores do serviço como: integração das ações de controle da hanseníase nos serviços, acesso facilitado à assistência, bem como a abordagem, a informação e a educação em saúde para a população. A verificação da maioria dos casos em idade produtiva amplia-se em importância considerando o potencial incapacitante da doença, o que gera diferentes impactos nos planos social, econômico, físico e psicológico (Monteiro et al., 2014).

No que diz respeito às formas clínicas, a forma indeterminada apresentou percentual baixo $(38,1 \%)$ em comparação a somatória das demais, denotando que a identificação das formas precoces ainda é precária. É importante ressaltar que, nesta fase, o diagnóstico adequado e o tratamento precoce interrompem a cadeia de transmissão. Interpreta-se, por meio dos dados, que os profissionais de saúde possuem dificuldades para classificá-las. Diante desta problemática, as sequelas causadas por esta doença afetam diretamente a qualidade de vida destas mulheres, uma vez que essas terão dificuldade nas atividades da 
vida diária, no próprio sustento e de sua família, além de gerar repercussões de ordem psicológica, social e física, comprometendo sua autoimagem feminina (Silva et al., 2018).

Um estudo desenvolvido entre 2009 e 2014 em centro de referência para hanseníase na Paraíba, verificou elevada porcentagem de indivíduos apresentando Grau I e Grau II tanto no diagnóstico (29,5\% e 11,1\%) quanto alta por cura (26,6\% e 10,9\%), demonstrando o caráter endêmico para a região Nordeste. A diminuição da ocorrência de casos novos com Grau 2 no momento do diagnóstico sugere redução do diagnóstico tardio conforme visto nas mulheres pesquisadas (Santana et al., 2018.).

Diante dessa premissa, no período pós-alta, a prevenção e o manejo de incapacidades residuais no período pós-alta ainda constituem um desafio, haja vista que após os pacientes concluírem o tratamento geralmente são excluídos do registro ativo e não recebendo, portanto, acompanhamento (Santana et al., 2018). Além disso, sobre as lacunas ou ausência de avaliação encontradas nos prontuários no que se refere ao GIF, evidenciando uma fragilidade do serviço, já que os dados da avaliação compõem os indicadores operacionais do Ministério da Saúde e mensuram a qualidade do atendimento nos serviços de saúde.

O conceito de cura do paciente com hanseníase deve ser ampliado para além da interrupção da transmissão, uma vez que há o risco potencial de desenvolver episódios reacionais no pós-alta. Essa população que está fora do registro ativo, deve ser alvo de ações específicas de monitoramento e atenção, porque o aumento do número de pacientes que são curados sem incapacidade física e a manutenção deste status deve ser indicador da melhoria da gestão e das condições de saúde da população (Souza et al., 2018).

Acrescido a isso, na maioria das regiões do mundo a incidência da hanseníase é maior nos homens quando comparados às mulheres, isso ocorre devido à exposição de fatores desencadeantes da doença. $\mathrm{O}$ que também pode ser explicado pelo fato das mulheres buscarem mais os serviços de saúde, se preocupar com a autoestima, observarem o corpo com frequência e diante da percepção de algo diferente buscar o serviço de saúde. Dessa forma, tem maiores chances de evitar instalação de futuras incapacidades físicas, uma vez que o diagnóstico e tratamento ocorrem de forma oportuna, além disso, considera-se que a mulher tem maior adesão ao tratamento (Oliveira et al., 2020).

Entre as mulheres investigadas, 90,5\% não estavam grávidas no momento do diagnóstico, o que já não preocupa, pois é sabido que a imunidade feminina está preservada nesse período, tendo passado a fase crítica do último trimestre da gestação e os três primeiros meses da lactação, quando ocorre redução da imunidade celular (Oliveira et al., 2011).

O período gravídico puerperal tem sido associado à elevada incidência de aparecimento dos primeiros sinais ou ao agravamento da hanseníase, sendo mais crítico o período compreendido entre o último trimestre da gestação e os três primeiros meses da lactação, por alterações hormonais, metabólicas e do sistema imune. Na gestação, ocorre supressão relativa da imunidade celular, principalmente no terceiro trimestre, levando ao desencadeamento ou à piora das reações hansênicas tipo 2 , havendo também risco de recidiva da doença (Teixeira et al., 2010).

Todas as mulheres do presente estudo com 60 anos ou mais, ou seja, que configura a parcela idosa do estudo relataram ter realizado mamografia, já metade da amostra que se enquadra na faixa etária de 19 a 59 anos relataram não ter realizado o exame de rastreamento através da mamografia no período pós-alta. Sabe-se que mulheres a partir dos 50 anos têm maior risco de desenvolver câncer de mama. O acúmulo de exposições ao longo da vida e as próprias alterações biológicas com o envelhecimento aumentam, de modo geral, esse risco (WHO, 2020; Brasil, 2020b). Deste modo, o exame de rastreamento através da mamografia é de suma importância e o mais eficaz para detecção precoce de câncer de mama, reduzindo significativamente a mortalidade por esta doença (Migowski et al., 2018).

No que se refere à importância do acompanhamento no pós-alta dessas mulheres, para a prevenção e detecção precoce do câncer do colo do útero, a maioria das mulheres encontravam-se em dia com a realização do exame, semelhante a outra pesquisa que apontou um percentual de $(97,2 \%)$ das participantes tinham realizado o exame citológico (Falcão et al., 2014). A 
vista disso é fundamental que seja realizado periodicamente o exame citopatológico oncótico, pois esse um método eficaz para identificação prévia de possíveis alterações citológicas que podem levar ao desenvolvimento do câncer (Nazaré et al., 2020).

No atual estudo, foram apresentadas dificuldades relevantes com relação à busca por atendimento de pós-alta, uma vez que algumas mulheres não aceitaram participar do estudo, outras não foram encontradas e algumas mudaram de município. Não basta manter um vínculo com o paciente para que este se sinta seguro para retornar quando detectar alguma alteração, fazse necessária a elaboração e aplicabilidade de protocolos de atendimento às pessoas que se encontram no momento do pós-alta (Monteiro et al., 2014).

\section{Considerações Finais}

As características demográficas e reprodutivas da população estudada foram: mulheres predominantemente pardas de baixa escolaridade (fundamental incompleto) e analfabetas, com idade acima dos 55 anos, que desempenham funções do lar e com renda de 1 salário-mínimo e meio, em relação aos aspectos clínicos 38,1\% foram diagnosticadas com forma clínica indeterminada e 66,7\% paucibacilares. Observou-se a necessidade de um acompanhamento especial para as mulheres no pósalta da hanseníase, uma vez que a grande maioria destas mulheres se encontram em idade reprodutiva. Neste sentido, é essencial promover um cuidado em busca de prepará-las para uma prática anticonceptiva segura e a prevenção de uma gestação de alto risco.

Salienta-se, também, a necessidade do reconhecimento da hanseníase como possível afecção dermatológica presente na gravidez, onde vários trabalhos destacam tal prevalência, mesmo ainda sendo marginalizada ou esquecida. Ações programáticas e pesquisa relacionados ao tema "gestação e hanseníase" necessitam ser contempladas, de modo a diminuir as complicações da hanseníase, ocasionadas pelo desconhecimento do diagnóstico e até mesmo por uma gravidez não planejada. É essencial que as UBS, possam vincular essa clientela aos serviços de planejamento familiar do serviço a fim de garantir um cuidado integral a essa população.

Observou-se como principal limitação deste estudo, a dificuldade de localização das mulheres, pois muitos endereços estavam desatualizados e a elevada rotatividade dos profissionais de saúde do município de Rio Largo-AL, principalmente os enfermeiros das Unidades Básicas de Saúde visitadas na pesquisa, com isso, consequentemente muitas mulheres não foram localizadas.

Nessa perspectiva, sugere-se para pesquisas futuras que sejam avaliados além das mulheres no pós-alta em hanseníase, investiguem-se os familiares, assim como, utilizem-se testes estatísticos que permitam uma análise aprofundada das variáveis estudadas. Possibilitando uma ampliada reflexão acerca da importância da saúde da mulher acometida por doença infectocontagiosa no contexto da atenção básica. As informações aqui descritas poderão contribuir para a prevenção, diagnóstico, tratamento, formação de grupos de autocuidado e cuidados holísticos no pós-alta das pessoas atingidas pela hanseníase, ao agregar novo enfoque para os profissionais de saúde e os serviços de saúde em relação a essa patologia.

\section{Referências}

Albuquerque, A. R. de., Silva, J. V. de M., Barreto, E. de O., Fraga, C. A. de C., Santos, W. O. Dos., Silva, M. S. M. da., Souza, C. D. F. de., \& SalesMarques, C. (2020). Epidemiological, temporal and spatial dynamics of leprosy in a municipality in northeastern Brazil (2008-2017): an ecological study. Revista da Sociedade Brasileira de Medicina Tropical, 53, e20200246. https://doi.org/10.1590/0037-8682-0246-2020.

Boigny, R. N., de Souza, E. A., Romanholo, H. S. B., de Araújo, O. D., de Araújo, T. M. E., Carneiro, M. A. G., Grijó, M. D. F., Henz, N. de L. F. B., Dos Reis, A. da S., Pinto, M. S. A. P., Barbosa, J. C., \& Ramos, A. N. (2019). Persistence of leprosy in household social networks: Overlapping cases and vulnerability in endemic regions in Brazil. Cadernos de Saúde Pública, 35(2), 1-14. https://doi.org/10.1590/0102-311x00105318.

Cabral-Miranda, W., Chiaravalloti Neto, F., \& Barrozo, L. V. (2014). Socio-economic and environmental effects influencing the development of leprosy in Bahia, north-eastern Brazil. Tropical Medicine and International Health, 19(12), 1504-1514. https://doi.org/10.1111/tmi.12389. 
Capp, E., \& Nienov, O. H. (Orgs). (2020). Bioestatística quantitativa e aplicada. Universidade Federal do Rio Grande do Sul. Faculdade de Medicina. Programa de Pós-Graduação em Ciências da Saúde: Ginecologia e Obstetrícia. Porto Alegre: UFRGS. https://lume.ufrgs.br/bitstream/handle/10183/213116/001117616.pdf?sequence=1\&isAllowed=y.

Departamento de Vigilância das Doenças Transmissíveis, Secretaria de Vigilância em Saúde, Ministério da Saúde. Diretrizes para vigilância, atenção e eliminação da hanseníase como problema de saúde pública. Manual técnico-operacional. http://portalms.saude.gov. br/images/pdf/2017/novembro/22/GuiaPra tico-de-Hanseniase-WEB.pdf.

Falcão, G. B., Ibiapina, F. L. P., Feitosa, H. N., Feitosa, T. S., Lacerda, P. D. de, Braga, J. U., \& Carvalho, F. H. C. (2014). Fatores associados à realização de citologia para prevenção de câncer do colo uterino em uma comunidade urbana de baixa renda. Cadernos Saúde Coletiva, 22(2), 165-172. https://doi.org/10.1590/1414-462x201400020009.

Gonçalves, M., Prado, M. A. R. do, Silva, S. S. da, Santos, K. da S., Araujo, P. N. de, \& Fortuna, C. M. (2018). Work and Leprosy: women in their pains, struggles and toils. Revista Brasileira de Enfermagem, 71, 660-667. https://doi.org/10.1590/0034-7167-2017-0598.

Lopes, V. A. S., \& Rangel, E. M. (2014). Hanseníase e vulnerabilidade social: uma análise do perfil socioeconômico de usuários em tratamento irregular Saúde em Debate, 38(103), 817-829. https://doi.org/10.5935/0103-1104.20140074.

Martins-Melo, F. R., Ramos, A. N., Alencar, C. H., \& Heukelbach, J. (2016). Mortality from neglected tropical diseases in Brazil, 2000-2011. Bulletin of the World Health Organization, 94(2), 103-110. https://doi.org/10.2471/blt.15.152363.

Mieras, L. F., Anand, S., Van Brakel, W. H., Hamilton, H. C., Martin Kollmann, K. H., Mackenzie, C., Mason, I., \& Wickenden, A. (2015). Neglected Tropical Diseases, Cross-Cutting Issues Workshop, 4-6 February 2015, Utrecht, the Netherlands: Meeting report. International Health, 8 (February 2015), i7i11. https://doi.org/10.1093/inthealth/ihw001.

Migowski, A., Azevedo E Silva, G., Dias, M. B. K., Diz, M. D. P. E., Sant'Ana, D. R., \& Nadanovsky, P. (2018). Guidelines for early detection of breast cancer in Brazil. II - New national recommendations, main evidence, and controversies. Cadernos de Saúde Pública, 34(6), 1-15. https://doi.org/10.1590/0102-311X00074817.

Ministério da Saúde. Boletim epidemiológico: hanseníase. Brasília: Ministério da saúde. http://www.aids.gov.br/pt-br/pub/2020/boletim-epidemiologico-dehanseniase-2020.

Ministério da Saúde. Instituto Nacional do Câncer - INCA. Estimativas da incidência e mortalidade por câncer. https://www.inca.gov.br/controle-do-cancerde-mama/fatores-de-risco .

Ministério da Saúde. Política nacional de saúde integral da população negra: uma política para o SUS. Brasília: Ministério da saúde. https://bvsms.saude.gov.br/bvs/publicacoes/politica_nacional_saude_populacao_negra_3d.pdf.

Monte, R. S., \& Pereira, M. L. D. (2015). Hansen's disease: social representations of affected people. Revista da Rede de Enfermagem do Nordeste, 16(6), 863. https://doi.org/10.15253/2175-6783.2015000600013.

Monteiro, L. D., Alencar, C. H., Barbosa, J. C., Novaes, C. C. B. S., da Silva, R. de C. P., \& Heukelbach, J. (2014). Pós-alta de hanseníase: Limitação de atividade e participação social em área hiperendêmica do Norte do Brasil. Revista Brasileira de Epidemiologia, 17(1), 91-104. https://doi.org/10.1590/1415790X201400010008ENG

Nazaré, G. de C. B., Ribeiro, J. C., Santos, A. A. dos, Resende, J. D. de S. A., Resende, M. A., \& Rodrigues, M. de S. (2020). A importância da busca ativa do enfermeiro na Atenção Primária para prevenção Do Câncer De Colo Uterino. Revista Eletrônica Acervo Saúde, 39 , e2066. https://doi.org/10.25248/reas.e2066.2020

Oliveira, A. E. V. M. de, Araújo, K. M. da F. A., Queiroga, R. P. F. de., Bezerra, L. L. O., \& Chaves, A. E. P. (2020). Análise epidemiológica da hanseníase por sexo na Paraíba. Research, Society and Development, 9(8), 1-15. https://doi.org/10.33448/rsd-v9i8.5778.

Oliveira, S. G. de, Tavares, C. M., Moura, E. R. F., Trindade, R. F. C., Almeida, A. M., \& Bomfim, E. de O. (2011). Gestação e hanseníase: uma associação de risco nos serviços de saúde. Hansenologia Internationalis, 36(1), 31-38. 01003283. http://periodicos.ses.sp.bvs.br/scielo.php?script=sci_arttext\&pid=S1982$51612011000100005 \& \operatorname{lng}=$ pt\&tlng=pt.

Santana, E. M. F. de, Brito, K. K. G. de, Nogueira, J. de A., Leadebal, O. D. C. P., Costa, M. M. L., Silva, M. A. da, \& Soares, M. J. G. O. (2018). Deficiências e incapacidades na hanseníase: do diagnóstico à alta por cura. Revista Eletrônica de Enfermagem, 20. https://doi.org/10.5216/ree.v20.50436.

Sarkar, R., \& Pradhan, S. (2016). Leprosy and women. International Journal of Women's Dermatology, 2(4), 117-121. doi: https://doi.org/10.1016/j.ijwd.2016.09.001.

Silva, C. M. D., Alves, R. S., Santos, T. S. D., Bragagnollo, G. R., Tavares, C. M., \& Santos, A. A. P. D. (2018). Epidemiological overview of HIV/AIDS in pregnant women from a state of northeastern Brazil. Revista Brasileira de Enfermagem, 71. https://doi.org/10.1590/0034-7167-2017-0495.

Souza, E. A. de, Ferreira, A. F., Boigny, R. N., Alencar, C. H., Heukelbach, J., Martins-Melo, F. R., Barbosa, J. C., \& Ramos, A. N. (2018). Leprosy and gender in Brazil: Trends in an endemic area of the Northeast region, 2001-2014. Revista de Saúde Pública, 52, 1-12. https://doi.org/10.11606/S15188787.2018052000335 .

Teixeira, M. A. G., da Silveira, V. M., \& de França, E. R. (2010). Characteristics of leprosy reactions in paucibacillary and multibacillary individuals attended at two reference centers in Recife, Pernambuco. Revista da Sociedade Brasileira de Medicina Tropical, 43(3), 287-292. https://doi.org/10.1590/s003786822010000300015 .

World Health Organization. Breast cancer: prevention and control. 2020. http://www.who.int/cancer/detection/breastcancer/en/.

World Health Organization. Global Leprosy Strategy 2016-2020: Accelerating towards a leprosy-free world. 2016. https://www.who.int/lep/resources/9789290225096/en/. 